\title{
Accurate determination of antenna directivity
}

\author{
Dich, Mikael
}

Published in:

I E E E Transactions on Antennas and Propagation

Link to article, DOI:

10.1109/8.633857

Publication date:

1997

Document Version

Publisher's PDF, also known as Version of record

Link back to DTU Orbit

Citation (APA):

Dich, M. (1997). Accurate determination of antenna directivity. I E E E Transactions on Antennas and Propagation, 45(10), 1502-1503. https://doi.org/10.1109/8.633857

\section{General rights}

Copyright and moral rights for the publications made accessible in the public portal are retained by the authors and/or other copyright owners and it is a condition of accessing publications that users recognise and abide by the legal requirements associated with these rights.

- Users may download and print one copy of any publication from the public portal for the purpose of private study or research.

- You may not further distribute the material or use it for any profit-making activity or commercial gain

- You may freely distribute the URL identifying the publication in the public portal

If you believe that this document breaches copyright please contact us providing details, and we will remove access to the work immediately and investigate your claim. 


\title{
Accurate Determination of Antenna Directivity
}

\author{
Mikael Dich, Member, IEEE
}

\begin{abstract}
The derivation of a formula for accurate estimation of the total radiated power from a transmitting antenna for which the radiated power density is known in a finite number of points on the far-field sphere is presented. The main application of the formula is determination of directivity from power-pattern measurements. The derivation is based on the theory of spherical wave expansion of electromagnetic fields, which also establishes a simple criterion for the required number of samples of the power density. An array antenna consisting of Hertzian dipoles is used to test the accuracy and rate of convergence of the derived formula. Its performance is compared to that of numerical integration using Simpson's formula.
\end{abstract}

Index Terms-Antenna theory.

\section{INTRODUCTION}

$\mathbf{T}$ HE directivity of a transmitting antenna is defined as $4 \pi$ times the ratio of the far-field power density to the total radiated power. The radiated power can be found directly by integrating the measured values of the power density over the entire sphere. Methods using numerical integration to obtain the total power from a set of power patterns are suggested in the literature [1], [2].

If the antenna measurement setup allows a determination of the tangential components of the radiated field over the entire sphere the field can be expanded into a sum of orthogonal spherical modes. The total power can be found by summing the power of all modes. This method is known to give accurate result [3], but the prescribed expansion can only be found if the field is measured in both amplitude and phase. However, as will be demonstrated it is also possible to expand the function describing the power density as a function of the spherical coordinates $\theta$ and $\phi$. From such an expansion a simple and accurate formula for the total radiated power can be derived.

\section{Determination of Directivity}

The directivity $D$ of a transmitting antenna in the direction $(\theta, \phi)$ is defined as

$$
D(\theta, \phi)=4 \pi \frac{P(\theta, \phi)}{P_{\text {tot }}}
$$

where the function $P(\theta, \phi)$ denotes the radiated power per unit solid angle and $P_{\text {tot }}$ the total radiated power. This definition does not take into account any dissipation or reflection losses of the antenna. The power function $P$ is related to the electrical

Manuscript received November 11, 1996.

The author is with the Department of Electromagnetic Systems, Technical University of Denmark, Lyngby, 2800 Denmark

Publisher Item Identifier S 0018-926X(97)07217-7. far field as

$$
P(\theta, \phi)=\frac{1}{2 \eta}\left[\left|E_{\theta}(\theta, \phi)\right|^{2}+\left|E_{\phi}(\theta, \phi)\right|^{2}\right]
$$

where $\eta=\sqrt{\mu_{0} / \varepsilon_{0}}$ is the intrinsic impedance of free-space.

The total radiated power can be found by integrating the power function

$$
\begin{aligned}
P_{\text {tot }} & =\int_{0}^{\pi}\left[\int_{0}^{2 \pi} P(\theta, \phi) d \phi\right] \sin \theta d \theta \\
& =\int_{0}^{\pi} P(\theta) \sin \theta d \theta \\
P(\theta) & =\int_{0}^{2 \pi} P(\theta, \phi) d \phi .
\end{aligned}
$$

An exact evaluation of the power integral (3) requires a complete knowledge of the power function $P(\theta, \phi)$ over the entire sphere. As described by Silver [1] and Tai and Pereira [2], the $\phi$ integration in (5) can be approximated by $2 \pi$ times the average of the power function in the four half-planes $\phi=0, \pi / 2, \pi$, and $3 \pi / 2$. This should work fairly well for pencil-beam antennas pointing in the direction of the $z$ axis. However, the accuracy of the approximation is not easily evaluated and even with this simplification one has yet to choose a method for the calculation of the $\theta$ integral in (4).

\section{FOURIER EXPANSION OF THE POWER FunCTION}

From the theory of spherical wave expansions (SWE) [3], it is known that the electric field radiated from an antenna can be described by

$$
\mathbf{E}(r, \theta, \phi)=k \sqrt{\eta} \sum_{s=1}^{2} \sum_{n=1}^{N} \sum_{m=-n}^{n} Q_{s m n}^{(3)} \mathbf{F}_{s m n}^{(3)}(r, \theta, \phi)
$$

where $k$ is the wave number, $Q_{s m n}^{(3)}$ are the coefficients of the expansion, and $\mathbf{F}_{s m n}^{(3)}$ the spherical vector wave functions (also referred to as spherical modes). The subscript $s=1,2$ denotes whether the function in question is TE or TM relative to $\hat{r}$.

Only a finite number of spherical modes is needed for an almost exact representation of the radiated field. The number of modes necessary is determined by the dimension of the antenna and the upper limit $N$ in the summation in (6) is given by

$$
N=\left[\frac{2 \pi r_{0}}{\lambda}\right]+n_{1}
$$

where the square brackets indicate the integer part and $r_{0}$ is the radius of the smallest sphere which is centered at the origin of the spherical coordinate system and completely encloses the 
antenna. The integer $n_{1}$ depends on the actual antenna and the required accuracy. However, $n_{1}=10$ is sufficient for all practical purposes.

For constant $r$ each of the orthogonal components $\mathbf{F}_{s m n}^{(3)} \cdot \hat{r}$, $\mathbf{F}_{s m n}^{(3)} \cdot \hat{\theta}$, and $\mathbf{F}_{s m n}^{(3)} \cdot \hat{\phi}$ of the spherical vector wave functions can be written as a Fourier series in $\theta$ and $\phi$. This implies that any of the tangential components $E_{\theta}(\theta, \phi)$ or $E_{\phi}(\theta, \phi)$ of the electric field can be written as

$$
E_{c}(\theta, \phi)=\sum_{n=-N}^{N} \sum_{m=-N}^{N} q_{c m n} e^{j n \theta} e^{j m \phi}
$$

where $c=\theta$ or $\phi$ and $q_{c m n}$ are scalar Fourier coefficients. Inserting (8) into (2) and noting that $|z|^{2}=z \cdot z^{*}$, we obtain a Fourier expansion of the power function (FEPF)

$$
P(\theta, \phi)=\sum_{n=-2 N}^{2 N} \sum_{m=-2 N}^{2 N} p_{m n} e^{j n \theta} e^{j m \phi}
$$

where $p_{m n}$ are the unknown Fourier coefficients. The essence of (9) is that for a given antenna, the power function $P(\theta, \phi)$ can be represented almost exactly by a finite Fourier expansion. The FEPF has approximately four times as many coefficients as the corresponding spherical wave expansion of the radiated field since the limits in (9) are twice the limits in (8). However, the power function is purely real so $p_{m n}=p_{-m,-n}=p_{-m, n}^{*}=p_{n,-m}^{*}$. Even though the FEPF in (9) can be used to expand the power function at any radius $r>r_{0}$ we will only use it in the case of the far field. Note that the far-field power function is unaffected by any translations of the antenna. The radius $r_{0}$ in (7) thus has a slightly different meaning compared to the case of the expansion of the far-field power function; in the latter case $r_{0}$ is just defined as the radius of the smallest sphere that encloses the antenna.

To find the total radiated power using the FEPF, we first consider the $\phi$ integration (5). For constant $\theta$ the power function $P(\theta, \phi)$ can be expressed as a Fourier series

$$
P(\theta, \phi)=\sum_{m=-2 N}^{2 N} p_{m}(\theta) e^{j m \phi}
$$

The coefficients $p_{m}$ can be found by first sampling $P(\theta, \phi)$ in at least $4 N+1$ equispaced points in the interval $0 \leq \phi<2 \pi$ and then applying the discrete Fourier transform

$$
p_{m}(\theta)=\frac{1}{L} \sum_{l=0}^{L-1} P\left(\theta, \frac{2 \pi l}{L}\right) e^{-j 2 \pi m l / L}, \quad L \geq 4 N+1 .
$$

The use of (10) and (11) in (5) yields

$$
P(\theta)=\frac{2 \pi}{L} \sum_{l=0}^{L-1} P\left(\theta, \frac{2 \pi l}{L}\right) .
$$

The $\phi$-integrated power function $P(\theta)$ can be expressed as a Fourier series similar to (10). However, if the permissible range of $\theta$ is extended into negative values by letting $P(-\theta, \phi)=P(\theta, \phi+\pi)$ we observe that $P(\theta)$ as defined by (12) is an even function. Therefore, it is more conveniently expressed as a cosine series

$$
P(\theta)=\sum_{n=0}^{2 N} p_{n} \epsilon(n) \cos (n \theta)
$$

where $\epsilon(n)$ is given by

$$
\epsilon(n)= \begin{cases}1 & \text { for } n=0 \\ 2 & \text { otherwise }\end{cases}
$$

The coefficients $p_{n}$ can be found by first sampling $P(\theta)$ in at least $2 N+2$ equispaced points in the interval $0 \leq \theta \leq \pi$ and then applying the discrete cosine transform

$$
\begin{gathered}
p_{n}=\frac{1}{4 K} \sum_{k=0}^{K} \epsilon(k) \epsilon(K-k) P\left(\frac{\pi k}{K}\right) \cos \left(\frac{\pi n k}{K}\right) \\
K \geq 2 N+1 .
\end{gathered}
$$

The use of (13) and (15) in (4) results in the following expression for the total radiated power:

$$
\begin{aligned}
P_{t o t}= & \frac{1}{4 K} \sum_{k=0}^{K} P\left(\frac{\pi k}{K}\right) \epsilon(k) \epsilon(K-k) \\
& \cdot \sum_{i=0}^{[K / 2]} \epsilon(i) \frac{2 \cos \left(\frac{2 \pi i k}{K}\right)}{1-4 i^{2}} \\
= & \frac{\pi}{2 K L} \sum_{k=0}^{K}\left[\sum_{l=0}^{L-1} P\left(\frac{\pi k}{K}, \frac{2 \pi l}{L}\right)\right] \\
& \epsilon(k) \epsilon(K-k) \sum_{i=0}^{[K / 2]} \epsilon(i) \frac{2 \cos \left(\frac{2 \pi i k}{K}\right)}{1-4 i^{2}} .
\end{aligned}
$$

This last formula, which we will refer to as the FEPF integration, can now be used in (1) to determine the directivity of an antenna. The spherical wave expansion (6) is highly accurate as long as $N$ is chosen according to (7). We can expect an accuracy of the same order in the determination of the total radiated power if $K$ and $L$ are chosen such that $K \geq 2 N+1$ and $L \geq 4 N+1$. These two requirements together with (7) constitute a criterion for the number samples needed. Note that if $L=2 K=4 N+2$ the sample spacing in $\theta$ and $\phi$ are the same.

\section{NUMERICAL RESULTS}

To investigate the accuracy and convergence properties of the FEPF integration (17), we will use an array antenna consisting of $x$-directed Hertzian dipoles, as shown in Fig. 1. The antenna is constructed of two planar arrays. The first is placed in the $x y$ plane at $z=0$. It consists of $15 \times 15$ dipoles spaced $\lambda / 2$ in both the $x$ and $y$ direction. The second planar array is identical to the first and is located at $z=-0.25 \lambda$. All 225 dipoles in the first array have the excitation $1=1 \angle 0$, whereas the 225 dipoles in the second array have the excitation $j=1 \angle 90^{\circ}$. The described antenna has maximum radiation in the $z$ direction and all sidelobes for $\theta>90^{\circ}$ are $26.5 \mathrm{~dB}$ or more below the main lobe. The radiation resembles that of 


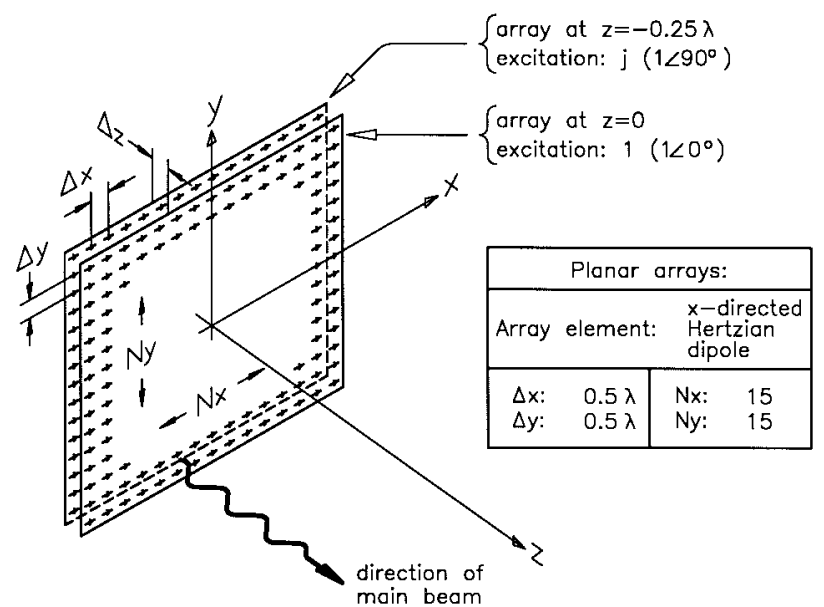

Fig. 1. Array antenna consisting of $x$-directed Hertzian dipoles. Radius of smallest sphere enclosing the antenna $\approx 5 \lambda$.

a planar array made of microstrip patches or waveguide fed slots where all elements have equal excitation. Even though an antenna with such a distribution of excitations has rather high sidelobes, it is not an uncommon choice in practical array antenna designs since the antennas hereby get the highest obtainable directivity.

The total radiated power from the array antenna has been calculated using two integration formulas. In the first case, the FEPF integration (17) was used. In the second case, the exact equation (12) was used for the $\phi$ integration and Simpson's formula was employed for the $\theta$ integration. The power $P_{\text {tot }}^{\text {simp }}$ is given by

$$
\begin{aligned}
P_{\text {tot }}^{\operatorname{simp}}= & \frac{2 \pi^{2}}{3 K} \sum_{k=0}^{K}\left[\sum_{l=0}^{L-1} P\left(\frac{\pi k}{K}, \frac{2 \pi l}{L}\right)\right] \sin (\theta) \\
& \cdot\left\{\begin{array}{ll}
1 & k=0 \text { and } K \\
2 & k=2,4, \cdots, K-2 \\
4 & k=1,3, \cdots, K-1
\end{array}\right\} .
\end{aligned}
$$

Due to the Simpson formula, the limit $K$ must be chosen evenly.

In Fig. 2, the error in percent of computed values of the radiated power is shown as a function of $N$. The solid line corresponds to the FEFP integration (17), while the dashed curve corresponds to the Simpson integration (18). In both cases the limits $L=4 N+2$ and $K=2 N+1$ have been used.

As can be seen, the accuracy of the FEPF integration is superior to that of the Simpson integration. The FEPF integration predicts the radiated power with an accuracy better than $1 \%$ for any $N>17$. One must choose $N>38$ to gain the same accuracy using the Simpson integration. To get a more clear picture of the rate of convergence, the logarithm of the absolute value of the relative error is show in Fig. 3. As can be seen, the error of the FEPF integration decreases extremely fast as $N$ is increased. For $N>30$ we have at least five significant digits of accuracy. In contrast, the error of the Simpson integration deceases slowly with $N$, yielding three significant digits of accuracy for $N>62$.

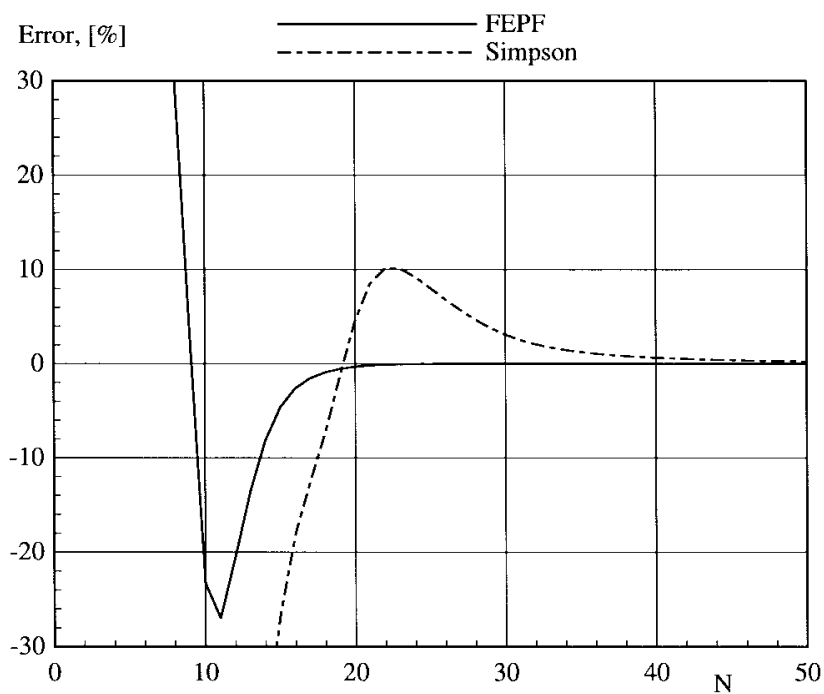

Fig. 2. Errors in percent of the computed values of radiated power as function of $N$. Solid line: Fourier expansion of power function (FEPF) integration. Dashed line: integration using Simpson's formula.

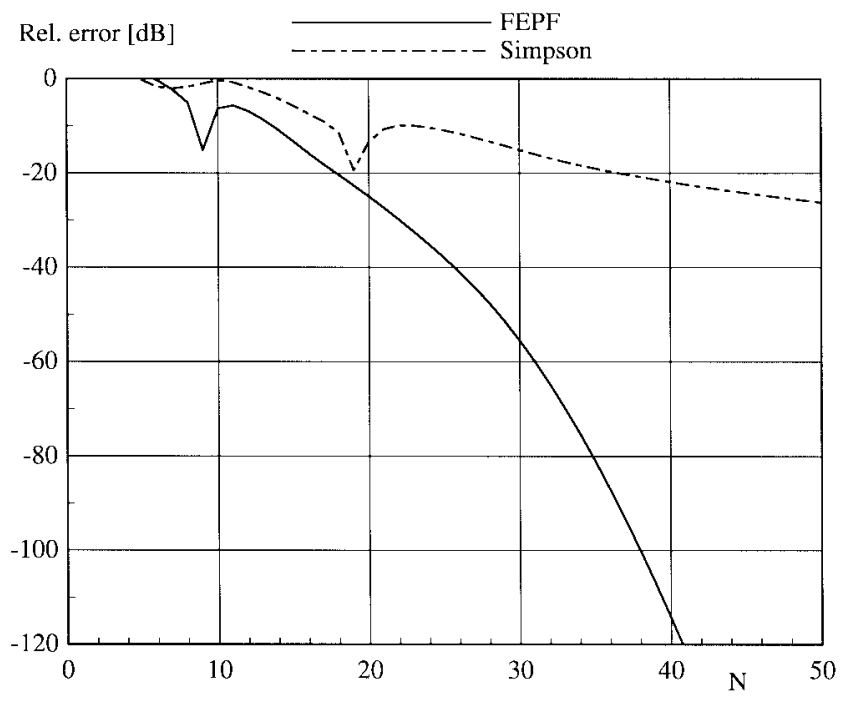

Fig. 3. $10 \log _{10}$ of the relative errors of the computed values of radiated power as function of $N$. Solid line: Fourier expansion of power function (FEPF) integration. Dashed line: integration using Simpson's formula.

The radius of the smallest sphere that encloses the antenna is approximately $5 \lambda$. From (7), we find that $N=41$ should give an almost exact value of the total radiated power using the FEPF integration. As seen from Fig. 3, $N=41$ gives indeed an extremely high accuracy for this idealized array antenna.

Finally, one could try the approximation suggested by Silver, and Tai and Pereira, i.e., replacing the $\phi$ integration (5) by $2 \pi$ times the average of the power functions in the four half-planes $\phi=0, \pi / 2, \pi$, and $3 \pi / 2$. This is the same as letting $L=4$ in (17) and (18). However, this approximation fails completely on the array antenna. The total radiated power is predicted $74 \%$ too high (both FEPF and Simpson's integration, $N=50$ ). This result, which could come as a surprise since this approximation is widely accepted, is due to a severe undersampling in $\phi$ of the power function $P(\theta, \phi)$. To investigate this, we interchange the order of integration in 


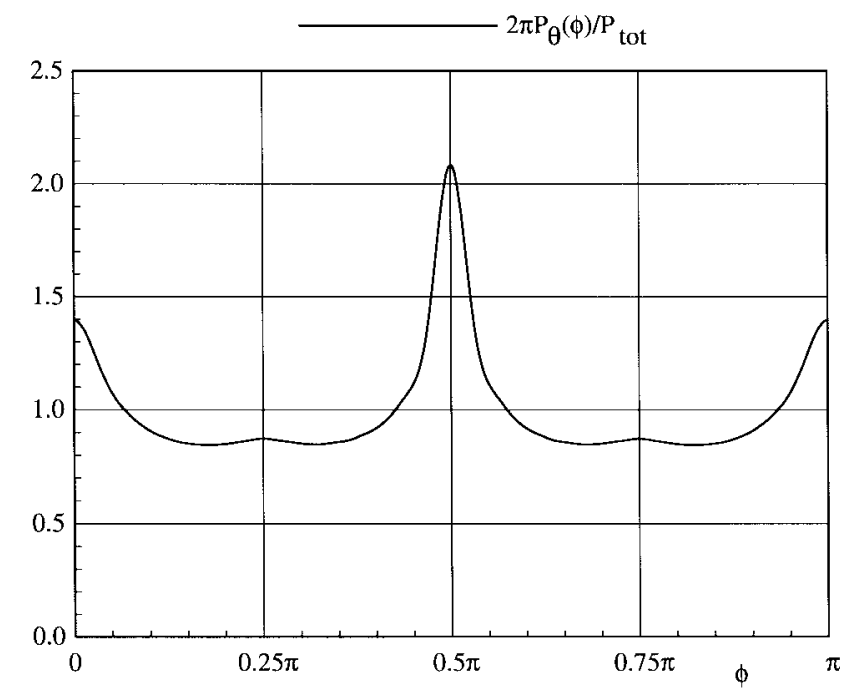

Fig. 4. The normalized function $P_{\theta}^{\text {no }}(\phi)=2 \pi P_{\theta}(\phi) / P_{\text {tot }}$ as a function of $\phi$. Note that $P_{\theta}^{\text {no }}(\phi)=P_{\theta}^{\text {no }}(\phi+\pi)$.

(3) yielding

$$
P_{\mathrm{tot}}=\int_{0}^{2 \pi} P_{\theta}(\phi) d \phi
$$

where

$$
P_{\theta}(\phi)=\int_{0}^{\pi} P(\theta, \phi) \sin \theta d \theta
$$

The total radiated power can now be found almost exactly by sampling $P_{\theta}(\phi)$ in at least $L=4 N+1$ equispaced points in the interval $0 \leq \phi<2 \pi$ and then applying the discrete integration

$$
P_{\text {tot }}=\frac{2 \pi}{L} \sum_{l=0}^{L-1} P_{\theta}\left(\frac{2 \pi l}{L}\right) .
$$

In Fig. 4 , the normalized function $P_{\theta}^{\text {no }}(\phi)=2 \pi P_{\theta}(\phi) /$ $P_{\text {tot, exact }}$ is shown as a function of $\phi$. The average value of the normalized function over the interval $0 \leq \phi<2 \pi$ is unity. The average of the four values $P_{\theta}^{\mathrm{no}}(0), \overline{P_{\theta}^{\mathrm{no}}}(\pi / 2), P_{\theta}^{\mathrm{no}}(\pi)$, and $P_{\theta}^{\mathrm{no}}(3 \pi / 2)$ is 1.74 corresponding to an error of $74 \%$ on the estimation of the total radiated power. As the number of samples is increased the average of the samples will get closer and closer to unity corresponding to a decrease of the error. The failure of the approximation is so pronounced since the sidelobes in the four half-planes $\phi=0, \pi / 2, \pi$, and $3 \pi / 2$ are substantially higher than in planes in between. For antennas with lower sidelobes the approximation can be expected to give a more reliable result. If the antenna possesses rotational symmetry about the $z$ axis the approximation becomes exact.

\section{CONCLUSION}

To determine the directivity of a transmitting antenna the total radiated power must be known. In this paper, a new formula for accurate determination of the total power is presented. The formula requires knowledge of the far-field power pattern over the entire sphere in a finite number of points equidistantly spaced in $\theta$ and $\phi$. The derived formula is simple and contains no references to any special functions.

A sampling criterion is also established. It specifies the required number of sample points on the sphere given the size of the antenna.

Using an array antenna consisting of Hertzian dipoles is has been demonstrated that for the same density of sample points the new formula determines the total radiated power more accurately than a formula based on simple numerical integration using Simpson's formula. It is also shown the new formula has far better convergence properties than the one using Simpson's formula.

\section{ACKNOWLEDGMENT}

The idea of carrying out the present investigation originated from F. Holm Larsen, deceased, who was the author's Ph.D. advisor.

\section{REFERENCES}

[1] S. Silver, Ed., Microwave Antenna Theory and Design. New York: McGraw-Hill, 1949, pp. 580-581.

[2] C.-T. Tai and C. S. Pereira, "An approximate formula for calculating the directivity of an antenna," IEEE Trans. Antennas Propagat., vol. AP-24, pp. 235-236, Mar. 1976.

[3] J. E. Hansen, Ed., Spherical Near-Field Antenna Measurements. London, U.K.: Peter Peregrinus, 1988.

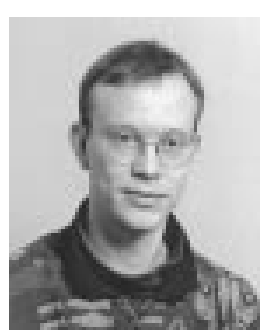

Mikael Dich (M'94) was born in Copenhagen, Denmark, on March 29, 1964. He received the M.S. and Ph.D. degrees in electronics from the Department of Electromagnetic Systems (EMI), Technical University of Denmark, in 1988 and 1994, respectively.

He has worked at EMI since 1988 with antennas, antenna measurements, numerical methods for electromagnetic problems, system software, and digital electronics. His main interests are slot antennas, antenna measurements, and numerical methods. 